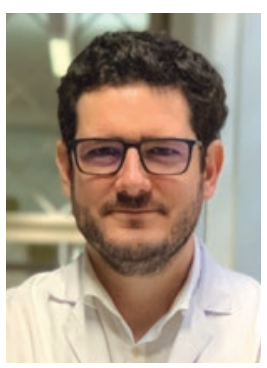

\title{
A debate: Intervención sobre las válvulas mitral y tricúspide. Perspectiva desde la cirugía
}

\author{
Debate: Intervention on the mitral and the tricuspid \\ valves. Perspective from surgery
}

\author{
Daniel Pereda $a, b, c, *$ \\ a Servicio de Cirugía Cardiovascular-Instituto Clínico Cardiovascular, Hospital Clínic, Barcelona, España \\ ${ }^{\mathrm{b}}$ Departamento de Cirugía y Especialidades Médico-quirúrgicas, Universitat de Barcelona, Barcelona, España

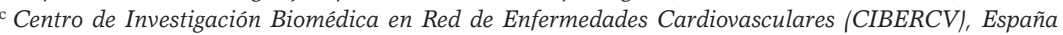

VÉASE CONTENIDO RELACIONADO:

https://doi.org/10.24875/RECIC.M21000208

Pregunta: ¿Cuál es el estado actual de la cirugía para la insuficiencia mitral (IM) funcional?

ResPuesta: La IM funcional es una de las condiciones clínicas de la cardiología que ha experimentado más cambios en los últimos años. No solo está en estudio la mejor manera de tratarla, incluyendo las aportaciones de las técnicas percutáneas, sino que hasta su propia definición está en revisión. Inicialmente se seleccionaron unos puntos de corte para definir su gravedad distintos que en la IM primaria, en función del resultado de estudios longitudinales en poblaciones tras un infarto de miocardio ${ }^{1}$. Sin embargo, ha sido difícil confirmar que las actuaciones terapéuticas usando estos umbrales de diagnóstico produzcan beneficios consistentes ${ }^{2,3}$ y actualmente se prefiere emplear los mismos criterios que en la IM primaria (orificio regurgitante efectivo $\geq 0,4 \mathrm{~cm}^{2}$ y volumen regurgitante $\geq 60 \mathrm{ml}$.

Otro aspecto de debate es si la mejor estrategia de tratamiento es la reparación o la sustitución de la válvula. La mayoría de los ensayos han evaluado la estrategia de reparación más simple, es decir, la anuloplastia restrictiva. Consiste en el remodelado anular con un anillo rígido o semirrígido, una o dos tallas menor que el tamaño de la válvula del paciente (normalmente determinada por la distancia intertrigonal o la superficie del velo anterior). A pesar de haber recibido críticas por la metodología usada en el grupo de reparación y por los resultados obtenidos tras la intervención, varios ensayos clínicos realizados en los Estados Unidos no han demostrado beneficios claros de la reparación sobre la sustitución valvular, aunque se ha observado una menor recurrencia de la IM en el grupo tratado con prótesis ${ }^{4,5}$. Muchos autores han señalado que este método de reparación no es el óptimo, principalmente si la etiología es isquémica, cuando el daño y el remodelado miocárdico, auténtico causante del problema valvular, suele ser localizado y muy variable.

Mi impresión es que se está llegando a cierta madurez en este campo. Parece claro que no hay una forma fácil de tratar a todos los pacientes y que el éxito depende de una adecuada selección de los candidatos y de las técnicas, incluyendo en particular el uso de técnicas sobre el aparato subvalvular, como el reposicionamiento o el cerclaje de los músculos papilares (papillary muscle sling). La IM secundaria es una enfermedad del ventrículo; esto lo decimos todos, pero la mayoría de los cirujanos y cardiólogos seguimos intentando solucionarla actuando únicamente sobre la válvula.

P.: ¿Qué técnicas son hoy las más adecuadas para la reparación de la válvula mitral con daño orgánico?

R.: El mejor tratamiento para la IM primaria es la reparación valvular quirúrgica, siempre que sea posible. Es el caso de casi la totalidad de los pacientes con degeneración mixoide tratados en centros con experiencia. También es la terapia de elección en otras etiologías, como la endocarditis y la enfermedad reumática, aunque con una menor tasa de reparación, dependiendo de las lesiones asociadas en la válvula y en el resto de las estructuras.

Es muy conocida la relación entre la reparación de la IM primaria y la experiencia del centro donde es tratada ${ }^{6}$. Sin embargo, esta experiencia es difícil de conseguir y la realidad es que hasta un $50 \%$ de los pacientes que podrían ser tratados mediante reparación continúan recibiendo una sustitución valvular ${ }^{7}$. Esto abre el debate sobre si los pacientes deben seguir unos patrones específicos de derivación a centros de excelencia en reparación mitral, donde se garanticen unos resultados óptimos, principalmente los pacientes jóvenes y en fases iniciales de la enfermedad (asintomáticos, en ritmo sinusal y con ventrículo izquierdo normal), como recomiendan las actuales guías clínicas ${ }^{8,9}$.

P.: ¿Existen buenas opciones quirúrgicas para la calcificación del anillo mitral?

R.: La calcificación anular es relativamente frecuente en la enfermedad reumática y en casos avanzados de degeneración mixoide,

\footnotetext{
* Autor para correspondencia: Cirugía Cardiovascular, Hospital Clínic, Villarroel 170, 08036 Barcelona, España. Correo electrónico: dpereda@clinic.cat (D. Pereda). 
y en general no supone un problema importante. Sin embargo, una minoría de pacientes presentan una calcificación muy extensa del anillo valvular, con infiltración profunda y extensión al tejido del miocardio ventricular. Son estos casos los que suponen un problema de solución muy compleja. La descalcificación incompleta del anillo conlleva una menor probabilidad de obtener una reparación exitosa y un mayor riesgo de un resultado subóptimo, con independencia de la técnica empleada: IM residual y estenosis asociada en el caso de la reparación, o fugas valvulares periprotésicas y un tamaño insuficiente de la prótesis en la sustitución valvular.

La solución a este problema es la realización de una descalcificación extensa, que es un procedimiento complejo y no exento de riesgos. Normalmente implica el desbridamiento agresivo del surco auriculoventricular posterior y su reconstrucción con algún tipo de material de refuerzo (pericardio autólogo o heterólogo, o tejidos sintéticos como el dacrón). Este tipo de reconstrucción requiere una adecuada selección de los pacientes y una técnica muy precisa, ya que su principal complicación, la rotura del surco posterior, asocia una muy elevada morbimortalidad.

Una interesante opción reciente, aunque con escasa información sobre sus resultados a medio plazo, es la combinación de un abordaje miniinvasivo para preparar la válvula y el implante bajo visión directa de una prótesis expandible, optimizando la fijación de la válvula y evitando la obstrucción del tracto de salida izquierdo.

P.: ¿Cuál es su opinión respecto a las técnicas quirúrgicas menos invasivas para la IM?

R.: Creo que en la actualidad son el método de elección para el tratamiento quirúrgico de la válvula mitral, ya que aportan ventajas evidentes. Muy recientemente publicamos en Revista Española de Cardiología $^{10}$ nuestra experiencia en el tratamiento del prolapso mitral, con una tasa de reparación exitosa del $98 \%$ y una mortalidad perioperatoria inferior al $1 \%$. La cirugía miniinvasiva toracoscópica fue nuestro abordaje preferido (más del $70 \%$ de los casos en los últimos años), que asocia menos ventilación mecánica, una estancia más corta en la unidad de cuidados intensivos, menor pérdida hemática y mayor ausencia de sustitución valvular a 5 años $(100 \%$ frente a $95 \%$ |

En noviembre de 2019 iniciamos nuestro programa de cirugía robótica, el único en España, empleando el sistema Da Vinci (Intuitive Surgical, Estados Unidos) y fundamentalmente centrado en la reparación mitral. Al momento de escribir estas líneas ya hemos intervenido a 50 pacientes, con resultados muy satisfactorios. En nuestra experiencia inicial consideramos que el procedimiento es incluso menos agresivo que la intervención toracoscópica y asocia una reducción adicional de la estancia posoperatoria, que de mediana es de 4 días.

No tengo ninguna duda de que estas técnicas miniinvasivas seguirán su expansión y refinamiento, y de que cada vez más pacientes se podrán beneficiar de una reparación quirúrgica con una menor agresión y una recuperación más rápida. En los próximos años aparecerán nuevas plataformas de cirugía robótica para uso clínico y continuará el desarrollo de opciones para el implante transapical de neocuerdas sin circulación extracorpórea. Estos avances ampliarán las opciones actuales de tratamiento y se incorporarán, junto con las técnicas percutáneas, a nuestro repertorio.

P.: La insuficiencia tricuspídea (IT) es una condición compleja de manejo frecuentemente no satisfactorio. ¿Qué puede ofrecer hoy día la cirugía y a quién?

R.: La IT ha ido recibiendo de manera progresiva la atención que merece. Uno de los cambios más importantes en los últimos años es la confirmación de la importancia de tratarla de forma precoz, incluso de manera profiláctica, aunque no exista, en el momento de intervenir una valvulopatía izquierda ${ }^{8,9}$. Esto se une al reconocimiento de la necesidad de tratar aisladamente la IT primaria grave en los pacientes con síntomas de insuficiencia cardiaca derecha y en aquellos asintomáticos con deterioro progresivo del ventrículo derecho ${ }^{9}$.

$\mathrm{Al}$ igual que en la IM secundaria, el tratamiento de la IT funcional requiere comprender el remodelado del ventrículo derecho y las lesiones no valvulares asociadas. Con demasiada frecuencia se ha tratado quirúrgicamente el problema de la IT secundaria de forma algo superficial, sin prestar el mismo grado de atención y detalle que en las valvulopatías izquierdas. Al igual que con la reparación mitral, el tratamiento de la IT secundaria requiere un importante nivel de formación y experiencia. En los centros de excelencia, los resultados de la reparación son excelentes y en ocasiones se requiere el uso de técnicas adicionales sobre los velos (ampliación del velo anterior con parche), el aparato subvalvular (reposicionamiento papilar, acortamiento o transposición de cuerdas, o implante de neocuerdas), el ventrículo derecho (plicatura de la pared libre y del anillo valvular) y la aurícula derecha. Estas técnicas menos frecuentes permiten extender los beneficios de la reparación a casos de IT funcional muy avanzados, etiologías primarias $y$, si se trata de una IT secundaria, a daños por cables de dispositivos o traumatismos torácicos. Un buen ejemplo de estos procedimientos más complejos y avanzados es la técnica de Da Silva de reparación valvular de pacientes adultos con anomalía de Ebstein ${ }^{11}$.

El tratamiento de la válvula tricúspide aislada o concomitante a la cirugía mitral también se puede realizar de forma miniinvasiva toracoscópica y robótica en centros con experiencia en la mayoría de los pacientes, e igualmente puede asociarse la ablación concomitante de la fibrilación auricular.

P.: ¿Cómo percibe el futuro de la cardiología intervencionista respecto a la cirugía cardiaca? ¿Hacia dónde debemos ir, tanto desde una especialidad como desde la otra?

R.: Personalmente creo que el futuro es brillante para los pacientes (lo más importante), los cirujanos cardiovasculares y los cardiólogos intervencionistas. La magnitud de estas mejoras y la rapidez con que se extiendan dependerán de nuestra capacidad para adquirir el conocimiento y las habilidades necesarias, para coordinarnos y para colaborar.

Mi opinión es que la cirugía tiene que avanzar hacia el perfeccionamiento de la formación en reparación valvular y en técnicas de mínima agresión, de manera que más pacientes se beneficien de las mejores opciones disponibles. No podemos seguir manteniendo las bajas tasas actuales de reparación valvular en patología mitral. Junto con la cardiología, debemos reflexionar sobre los patrones actuales de derivación de pacientes y buscar fórmulas para que sean intervenidos (de forma quirúrgica o percutánea) en centros con experiencia, que garanticen su acceso a todas las opciones de tratamiento y donde todas las técnicas se realicen con resultados óptimos, basándose en la evidencia científica y en la auditoría y la comunicación de sus propios resultados.

\section{FINANCIACIÓN}

Sin fuentes de financiación.

\section{CONFLICTO DE INTERESES}

D. Pereda es proctor de cirugía mínimamente invasiva para Edwards Lifesciences. 


\section{BIBLIOGRAFÍA}

1. Grigioni F, Enriquez-Sarano M, Zehr KJ, Bailey KR, Tajik AJ. Ischemic mitral regurgitation: long-term outcome and prognostic implications with quantitative Doppler assessment. Circulation. 2001;103:1759-1764.

2. Michler RE, Smith PK, Parides MK, et al. Two-year outcomes of surgical treatment of moderate ischemic mitral regurgitation. N Engl J Med. 2016; 374:1932-1941.

3. Smith PK, Puskas JD, Ascheim DD, et al. Surgical treatment of moderate ischemic mitral regurgitation. N Engl J Med. 2014;371:2178-2188.

4. Acker MA, Parides MK, Perrault LP, et al. Mitral-valve repair versus replacement for severe ischemic mitral regurgitation. N Engl J Med. 2014;370:23-32.

5. Goldstein D, Moskowitz AJ, Gelijns AC, et al. Two-year outcomes of surgical treatment of severe ischemic mitral regurgitation. $N$ Engl J Med. 2016;374:344-353.

6. Anyanwu AC, Bridgewater B, Adams DH. The lottery of mitral valve repair surgery. Heart. 2010;96:1964-1967.
7. Iung B, Baron G, Butchart EG, et al. A prospective survey of patients with valvular heart disease in Europe: The Euro Heart Survey on Valvular Heart Disease. Eur Heart J. 2003;24:1231-1243.

8. Baumgartner H, Falk V, Bax JJ, et al. 2017 ESC/EACTS Guidelines for the management of valvular heart disease. Eur Heart J. 2017;38:2739-2791.

9. Otto CM, Nishimura RA, Bonow RO, et al. 2020 ACC/AHA Guideline for the Management of Patients With Valvular Heart Disease: A Report of the American College of Cardiology/American Heart Association Joint Committee on Clinical Practice Guidelines. Circulation. 2021;143:e72-e227.

10. Ascaso M, Sandoval E, Quintana E, Vidal B, Castellà M, Pereda D. Early and mid-term outcomes of mitral repair due to leaflet prolapse in a national referral center. Rev Esp Cardiol. 2021;74:462-4.

11. Fernández-Cisneros A, Ascaso M, Sandoval E, Pereda D. Cone repair for Ebstein's anomaly and atrial fibrillation ablation in an adult patient. Multimed Man Cardiothorac Surg. 2020. http://dx.doi.org/10.1510/mmcts. 2020.064 . 